\title{
THE DIRICHLET PROBLEM AT INFINITY FOR NONPOSITIVELY CURVED MANIFOLDS
}

\author{
SHIU-YUEN CHENG
}

\section{INTRODUCTION}

Let $M^{n}$ be an $n$-dimensional complete non-compact Riemannian manifold. The Dirichlet problem of harmonic functions with prescribed boundary data at infinity is a very interesting problem in geometry and analysis. In [S], using a probablistic approach, this problem was solved when $M$ is simply-connected and the sectional curvatures are bounded between two negative constants. Then, following the program of Choi, it was solved in [A] under the same assumptions by a more geometric approach. In this paper, we'll follow the program by Anderson and Schoen in [A-S]. In fact, the more general problem of the Martin boundary was solved in [A-S].

The approach of Anderson and Schoen is as follows: For a given boundary data, a smooth function $f$ on $M$ which assumes the boundary data is constructed explicitly. Then, on a geodesic ball $B\left(x_{0}, R\right)$ with center $x_{0}$ and radius $R$ solve

$$
\begin{cases}\Delta u_{R}=-\Delta f & \text { on } B\left(x_{0}, R\right) \\ u_{R}=0 & \text { on } \partial B\left(x_{0}, R\right) .\end{cases}
$$

Clearly, $u_{R}+f$ is harmonic on $B\left(x_{0}, R\right)$ and $u_{R}+f=f$ on $\partial B\left(x_{0}, R\right)$ : Then a harmonic function is obtained if one can extract a converging subsequence from $\left\{u_{R}+f\right\}$ by letting $R \rightarrow \infty$. For this purpose, a barrier function is constructed explicitly and it can be applied to (0.1) if $\Delta f$ is of exponential decay. A priori, $\Delta f$ will not satisfy this property. The ingenious observation in [A-S] is that all is well if $f$ is mollified first and then plucked into (0.1) to 
solve for $u_{R}$. To do the mollifying process, the assumptions that injectivity is bounded away from zero and bounded sectional curvatures are essential.

In [S-Y], Schoen and Yau then followed this approach to prove the following theorem with a much more elaborate construction of the barrier function.

Theorem (Schoen-Yau). Suppose $\left(M, d \tilde{s}^{2}\right)$ is complete, simply-connected and its sectional curvatures $\tilde{K}_{M}$ satisfy $-b^{2} \leq \tilde{K}_{M} \leq-a^{2}<0$. Let $d s^{2}$ be another metric on $M$ uniformly equivalent to $d \tilde{s}^{2}$, i.e. there exists $A>0$ so that $\frac{1}{A} d s^{2} \leq d \tilde{s}^{2} \leq A d s^{2}$. Suppose the sectional curvatures of $\left(M, d s^{2}\right)$ are bounded from above and below and that the injectivity radius of $\left(M, d s^{2}\right)$ is bounded away from zero. Then, for any continuous function $f$ on $M(\infty)$, there exists $u \in C^{\infty}(M) \cap C^{0}(M \cup M(\infty))$ so that

$$
\begin{cases}\Delta u=0 & \text { on } M \\ u=f & \text { on } M(\infty)\end{cases}
$$

where $\Delta$ is the Laplacian operator of $\left(M, d s^{2}\right)$.

The assumption that $d s^{2}$ is uniformly equivalent to $d \tilde{s}^{2}$ is very flexible but the assumptions on the curvatures and injectivity radius of $\left(M, d s^{2}\right)$ are somewhat restrictive. This paper was grown out from the attempt to remove the assumptions on the injectivity radius and sectional curvatures from the Schoen-Yau theorem. In fact, Ancona [Anc] proved that this indeed can be done. We'll prove this in Theorem 2.2. Our method uses more properties of $f$ so that we can avoid the use of the mollifying process. In Section 1, a key proposition is proved to estimate the $L^{p}$-norm of $u_{R}$ in terms of the $L^{p}$-norm of $|\nabla f|$. This estimate guarantees a subsequence of $\left\{u_{R}+f\right\}$ converges to a global harmonic function on $M$. In Section 2, the boundary value at infinity of the harmonic function constructed in Section 1 is discussed and a sub-meanvalue inequality is proved. In Section 3, we use the results in Section 1 and Section 2 to solve the Dirichlet problem at infinity for nonpositively curved manifolds satisfying a local pinching condition. This generalizes the results on rotationally symmetric manifolds studied by Choi in [C].

Acknowledgement. The author would like to thank Professors Peter Li and L. F. Tam for interesting and insightful discussions and suggestions. 


\section{An $L^{p}$-NORM ESTIMATE}

We will try to estimate the $L^{p}$-norm of $u_{R}$ in (0.1) in terms of the $L^{p}$-norm of $|\nabla f|$. In fact, one observes easily that both in [A-S] and [S-Y], $|\nabla f|$ can be assumed to be of exponential decay so that $\int|\nabla f|^{p}<+\infty$ for a large $p$. We'll use this property in the following proposition to estimate the $L^{p}$-norm of $u_{R}$.

Proposition 1.1. Let $M^{n}$ be an n-dimensional Riemannian manifold. Suppose also that $\lambda_{1}(M)>0$. Let $\Omega$ be any compact subdomain of $M$ such that $\partial \Omega$ is smooth. Let $f \in C^{\infty}(M)$ and $u$ be a solution of

$$
\begin{cases}\Delta u=-\Delta f & \text { on } \Omega, \\ u=0 & \text { on } \partial \Omega .\end{cases}
$$

Then, for any $p \geq 2$

$$
\int_{\Omega}|u|^{p} \leq C \int_{\Omega}|\nabla f|^{p}
$$

where $C$ is a positive constant depending only on $p$ and $\lambda_{1}(M)$.

Proof. Multiply $(\operatorname{sgn} u)|u|^{p-1}$ to (1.1) and integrate we get

$$
\int_{\Omega}|u|^{p-2}|\nabla| u||^{2} \leq \int_{\Omega}|u|^{p-2}|\nabla| u|||\nabla f| .
$$

Apply the Schwartz inequality to the right hand side of (1.3), we derive that

$$
\int_{\Omega}|u|^{p-2}|\nabla| u||^{2} \leq \int_{\Omega}|u|^{p-2}|\nabla f|^{2} .
$$

Therefore,

$$
\left.\left.\frac{4}{p^{2}} \int_{\Omega}|\nabla| u\right|^{\frac{p}{2}}\right|^{2} \leq\left(\int_{\Omega}|u|^{p}\right)^{\frac{p-2}{p}}\left(\int_{\Omega}|\nabla f|^{p}\right)^{\frac{2}{p}} .
$$

As $\lambda_{1}(\Omega) \geq \lambda_{1}(M)$ we have,

$$
\frac{4}{p^{2}} \lambda_{1}(M) \int_{\Omega}|u|^{p} \leq\left(\int_{\Omega}|u|^{p}\right)^{\frac{p-2}{p}}\left(\int_{\Omega}|\nabla f|^{p}\right)^{\frac{2}{p}} .
$$

Thus,

$$
\int_{\Omega}|u|^{p} \leq\left(\frac{p^{2}}{4 \lambda_{1}(M)}\right)^{\frac{p}{2}} \int_{\Omega}|\nabla f|^{p} .
$$

This proves the $L^{p}$-bound on $u$. 
Remark 1.1. Substituting (1.5) into (1.4), we get a bound on $\left.\left.\int_{\Omega}|\nabla| u\right|^{\frac{p}{2}}\right|^{2}$ in terms of the $L^{p}$-norm of $|\nabla f|$.

Now we can use Proposition 1.1 to obtain a converging subsequence from the solutions to $(0.1)$.

Theorem 1.2. Suppose $M$ as in Proposition 1.1, $f \in C^{\infty}(M)$ and $\int_{M}|\nabla f|^{p}<$ $+\infty$ for some $p \geq 2$. Let $u_{R}$ be the solutions of (0.1). Then a subsequence of $u_{R}$ will converge uniformly on compact subsets to $u \in C^{\infty}(M)$ such that $\Delta u=-\Delta f$ on $M$ and $\int_{M}|u|^{p}<+\infty$.

Proof. Using Proposition 1.1 we see that $\int_{B\left(x_{0}, R\right)}|u|^{p}$ is uniformly bounded independent of $R$. Then, $u_{R}+f$ is a harmonic function on $B\left(x_{0}, R\right)$ such that its $L^{p}$-norm is bounded independent of $R$. Now it is easy to finish the proof. We can use the sub-meanvalue inequality in [S-Y, Theorem 2.13] to $\left|u_{R}+f\right|$. Hence its supremum norm is bounded on compact subsets independent of $R$. It is then standard to see that the assertions of the Theorem are true.

Remark 1.2. The assumption that $\lambda_{1}(M)>0$ is a reasonable assumption in the problems of existence of harmonic functions. It is satisfied by a complete, simply-connected manifold with sectional curvatures bounded from above by a negative constant. Then any manifolds which is quasi-isometric to such a manifold also enjoys this property.

Remark 1.3. The assumption that $|\nabla f|$ is $L^{p}$ for some large $p$ is also satisfied in the Anderson-Schoen [A-S] paper as well as in the Schoen-Yau theorem.

\section{Boundary Values}

To show the harmonic function $u+f$ obtained in Theorem 1.2 assumes the boundary data, we need to show that $u(x) \rightarrow 0$ as $x \rightarrow \infty$. This will follow from $\int_{M}|u|^{p}<+\infty$ and additional assumptions on $M$ and $f$ so that the supremum norm of $u$ on geodesic balls can be estimated. One of the usual techniques is the gradient estimate.

Theorem 2.1. Suppose $M^{n}$ is a complete, non-compact n-dimensional Riemannian manifold such that $\lambda_{1}(M)>0$. Assume that, for any $r>0$, $\inf _{x \in M} \operatorname{Vol}(B(x, r))>0$, and the Ricci curvatures are bounded from below. 
Given a bounded smooth function $f$ on $M$ with $|\nabla f|$ bounded and $\int_{M}|\nabla f|^{p}<$ $+\infty$ for some $p \geq 2$, there exists $u \in C^{\infty}(M)$ so that $\Delta(u+f)=0$ on $M$, $\int_{M}|u|^{p}<+\infty$ and $u(x) \rightarrow 0$ as $x \rightarrow \infty$.

Proof. By adding a constant to $f$ if necessary, we may assume $f$ to be positive. Then Theorem 1.2 implies the existence of a function $u \in C^{\infty}(M)$ such that $\Delta(u+f)=0$ on $M$ and $\int_{M}|u|^{p}<+\infty$. Also $u_{R}+f$ is positive on $B\left(x_{0}, R\right)$ because it is positive on the boundary. As $u+f$ is the limit of a subsequence of $\left\{\left(u_{R}+f\right)\right\}$, we see that $u+f$ is a bounded and positive harmonic function on $M$. Using the gradient estimate in [Y], we see that $|\nabla \log (u+f)|$ is also bounded on $M$. Since $u+f$ and $|\nabla f|$ are bounded on $M$, we derive that $|\nabla u|$ is bounded on $M$. The rest of the proof then follows easily from the assumption on the volume of the geodesic balls and that $\int_{M}|u|^{p}<+\infty$.

Remark 2.1. Theorem 2.1 gives an alternative proof of solving the Dirichlet problem at infinity when $M$ is complete simply-connected and sectional curvatures bounded between two negative constants.

Another technique to prove $u(x) \rightarrow 0$ as $x \rightarrow \infty$ is to use the Moser iteration technique to prove a sub-meanvalue type inequality.

Theorem 2.2. Suppose that $M$ is a complete Riemannian manifold and there exists $S_{1}>0$ such that for any $\phi \in C_{0}^{\infty}(M)$ we have $S_{1}\left(\int|\phi|^{\kappa}\right)^{1 / \kappa} \leq \int|\nabla \phi|$, where $\kappa=n /(n-1)$. Suppose that $u$ is a bounded function in $L^{p}(M)$ satisfying $\Delta u=-\Delta f$, where $|\nabla f|$ is bounded and $|\nabla f| \in L^{p}$ for some $p \geq 2$. Then, there exists a constant $\delta \in(0,1)$ so that for any $z \in M, \tau_{0} \in(0,1)$ and $R_{0}>0$, we have for $R \in\left(0, R_{0}\right)$

$$
\sup _{B\left(z,\left(1-\tau_{0}\right) R\right)}|u|^{p} \leq \frac{C}{\tau_{0}^{n} R^{n}}\left(\int_{B(z, R)}|u|^{p}\right)^{\delta},
$$

where $C$ is a constant depending only on $n, p, R_{0}, \sup _{M}|u|, \sup _{M}|\nabla f|, \int|u|^{p}$ and $\int|\nabla f|^{p}$.

Proof. Without loss of gererality we may suppose that $|u| \leq 1$ and that $\int|u|^{p} \leq$ 1. Then let $\eta$ be a cut-off function to be specified later. Multiply both sides 
of the equation $\Delta u=-\Delta f$ by $\eta^{2}(\operatorname{sgn} u)|u|^{q-1}$ and then integrate we get

$$
\begin{aligned}
& -(q-1) \int \eta^{2}(\operatorname{sgn} u)|u|^{q-2} \nabla u \cdot \nabla|u|-2 \int|u|^{q-1} \eta(\operatorname{sgn} u) \nabla u \cdot \nabla \eta \\
& \quad=(q-1) \int \eta^{2}(\operatorname{sgn} u)|u|^{q-2} \nabla|u| \cdot \nabla f+2 \int \eta(\operatorname{sgn} u)|u|^{q-1} \nabla \eta \cdot \nabla f .
\end{aligned}
$$

Rearranging the terms and applying the Schwartz inequality we have

$$
\begin{aligned}
& \left.(q-1) \int \eta^{2}|u|^{q-2}|\nabla| u\right|^{2} \\
& \leq(q-1)\left(\int \eta^{2}|u|^{q-2}|\nabla| u||^{2}\right)^{1 / 2} \cdot\left(\int \eta^{2}|u|^{q-2}|\nabla f|^{2}\right)^{1 / 2} \\
& \quad 2 \int|u|^{q-1} \eta|\nabla| u|| \cdot|\nabla \eta|+2 \int|u|^{q-1} \eta|\nabla f| \cdot|\nabla \eta| .
\end{aligned}
$$

Hence,

$$
\begin{aligned}
\left.\frac{3}{4}(q-1) \int \eta^{2}|u|^{q-2}|\nabla| u\right|^{2} & \\
\leq(q-1) \int \eta^{2}|u|^{q-2}|\nabla f|^{2} & +2\left(\left.\int \eta^{2}|u|^{q-2}|\nabla| u\right|^{2}\right)^{1 / 2}\left(\int|u|^{q}|\nabla \eta|^{2}\right)^{1 / 2} \\
& +2\left(\int \eta^{2}|u|^{q-2}|\nabla f|^{2}\right)^{1 / 2}\left(\int|u|^{q}|\nabla \eta|^{2}\right)^{1 / 2} .
\end{aligned}
$$

Then,

$$
\left.\frac{(q-1)}{2} \int \eta^{2}|u|^{q-2}|\nabla| u\right|^{2} \leq 2(q-1) \int \eta^{2}|u|^{q-2}|\nabla f|^{2}+\frac{5}{q-1} \int|u|^{q}|\nabla \eta|^{2} .
$$

Note that

$$
\left.\left.|\nabla| u\right|^{\frac{q}{2}}\right|^{2}=\left.\frac{q^{2}}{4}|u|^{q-2}|\nabla| u\right|^{2} \leq\left.(q-1)^{2}|u|^{q-2}|\nabla| u\right|^{2}
$$

for $q \geq 2$. Therefore,

$$
\left.\left.\int \eta^{2}|\nabla| u\right|^{\frac{q}{2}}\right|^{2} \leq 4(q-1)^{2} \int \eta^{2}|u|^{q-2}|\nabla f|^{2}+10 \int|u|^{q}|\nabla \eta|^{2} .
$$

Also,

$$
\begin{aligned}
\left.\left.\left.\left|\nabla \eta^{2}\right| u\right|^{q}\left|\leq 2 \eta^{2}\right| u\right|^{\frac{q}{2}}|\nabla| u\right|^{\frac{q}{2}} \mid & +2 \eta|u|^{q}|\nabla \eta| \\
& \leq\left.\left.\epsilon \eta^{2}|\nabla| u\right|^{\frac{q}{2}}\right|^{2}+2 \epsilon^{-1} \eta^{2}|u|^{q}+\epsilon|u|^{q}|\nabla \eta|^{2}
\end{aligned}
$$

where $\epsilon$ is any positive number. Now we let $\eta(x)=\rho(r)$, where $r$ is the distance function from $z$ and $\rho$ is a piecewise linear function of one variable such that $\rho=1$ on $[0, \theta R], \rho=0$ on $[(\theta+\tau) R, \infty)$ and linear on $[\theta R,(\theta+\tau) R]$. Then, 
by setting $\epsilon=\tau R$ in (2.3) and combining (2.2), (2.3) and using the Sobolev inequality we derive that

$$
\begin{aligned}
S_{1}\left(\int_{B(z, \theta R)}|u|^{\kappa q}\right)^{1 / \kappa} \leq \frac{13}{\tau R} \int_{B(z,(\theta+\tau) R)}|u|^{q} & \\
& +4(q-1)^{2} \tau R \int_{B(z,(\theta+\tau) R)}|u|^{q-2}|\nabla f|^{2}
\end{aligned}
$$

Applying Hölder inequality to the last term of (2.4), it becomes

$$
\begin{aligned}
& \left(\int_{B(z, \theta R)}|u|^{\kappa q}\right)^{1 / \kappa} \leq \frac{13}{\tau R S_{1}} \int_{B(z,(\theta+\tau) R)}|u|^{q} \\
& +\frac{4(q-1)^{2} \tau R}{S_{1}}\left(\int_{B(z,(\theta+\tau) R)}|u|^{q}\right)^{\frac{q-2}{q}}\left(\int_{B(z,(\theta+\tau) R)}|\nabla f|^{q}\right)^{\frac{2}{q}} .
\end{aligned}
$$

Let $A=\sup |\nabla f|$. Then, clearly for $p \leq q$

$$
\int \frac{|\nabla f|^{q}}{A^{q}} \leq \int \frac{|\nabla f|^{p}}{A^{p}}
$$

So

$$
\left(\int|\nabla f|^{q}\right)^{2 / q} \leq A^{2(1-p / q)}\left(\int|\nabla f|^{p}\right)^{2 / q} .
$$

Using this in (2.5), we can rewrite (2.5) as

$$
\left(\int_{B(z, \theta R)}|u|^{\kappa q}\right)^{1 / \kappa} \leq \frac{C_{1}}{\tau R S_{1}}\left(1+(q-1)^{2} \tau^{2} R^{2}\right)\left(\int_{B(z,(\theta+\tau) R)}|u|^{q}\right)^{\frac{q-2}{q}}
$$

where $C_{1}$ is a constant depending only on the supremum and $L^{p}$ norm of $|\nabla f|$.

Now let us choose $q_{i}=p \kappa^{i}, \tau_{i}=\tau_{0} 2^{-i}, \theta_{0}=1$ and $\theta_{i+1}=\theta_{i}-\tau_{i+1}$. Set $\theta=\theta_{i+1}, \tau=\tau_{i+1}, q=q_{i}$ in (2.6) and observe that $\left(q_{i}-1\right) \tau_{i+1}$ is bounded from above independent of $i$ because $\kappa \leq 2$, we have

$$
\left(\int_{B\left(z, \theta_{i+1} R\right)}|u|^{\kappa q_{i}}\right)^{1 / \kappa} \leq \frac{C_{2}}{\tau_{i+1} R}\left(\int_{B\left(z, \theta_{i} R\right)}|u|^{q_{i}}\right)^{\frac{q_{i}-2}{q_{i}}}
$$

where $C_{2}$ is a constant depending only on $p, R_{0}, S_{1}$, the supremum and $L^{p}$ norm of $|\nabla f|$. 
Define $I_{i}=\left(\int_{B\left(z, \theta_{i} R\right)}|u|^{p \kappa^{i}}\right)^{1 / \kappa_{i}}$, then by taking $1 / \kappa^{i}$-th power of both sides of (2.7) we obtain

$$
I_{i+1} \leq 2^{(i+1) / \kappa^{i}}\left(\frac{C_{2}}{\tau_{0} R}\right)^{1 / \kappa^{i}} I_{i}^{\left(p \kappa^{i}-2\right) / p \kappa^{i}}
$$

(2.1) then follows by applying (2.8) recursively with $\delta=\prod\left(p \kappa^{i}-2\right) / p \kappa^{i}>$ 0 .

Now we can apply our method to generalize the Schoen-Yau theorem which is also proved by Ancona in [Anc].

Theorem 2.3. Suppose $\left(M, d \tilde{s}^{2}\right)$ is a complete, simply-connected $n$-dimensional Riemannian manifold such that its sectional curvatures $\tilde{K}_{M}$ satisfies $-b^{2} \leq \tilde{K}_{M} \leq-a^{2}<0$. Let $d s^{2}$ be a metric on $M$ uniformly equivalent to $d \tilde{s}^{2}$, i.e. there exists $A>0$ so that $\frac{1}{A} d s^{2} \leq d \tilde{s}^{2} \leq A d s^{2}$. Then for any continuous function $f$ on $M(\infty)$, there exists $u \in C^{\infty}(M) \cap C^{0}(M \cup M(\infty))$ so that

$$
\begin{cases}\Delta u=0 & \text { on } M \\ u=f & \text { on } M(\infty),\end{cases}
$$

where $\Delta$ is the Laplacian operator of $\left(M, d s^{2}\right)$.

Proof. Without loss of generality, we may assume that $f$ is positive and can be extended to $\left(M, d \tilde{s}^{2}\right)$ as a positive smooth function such that outside a compact subset it is radially constant with respect to some point $x_{0} \in M$. As in [A-S], using the curvature assumptions of $\left(M, d \tilde{s}^{2}\right)$, we see that $\left|\nabla_{d \tilde{s}^{2}} f\right|$ is of exponential decay and is in $L^{p}$ for a sufficiently large $p$. Since $\left(M, d s^{2}\right)$ is uniformly equivalent to $\left(M, d \tilde{s}^{2}\right),\left|\nabla_{d s^{2}} f\right|$ is also of exponential decay and in $L^{p}$ with respect to $\left(M, d s^{2}\right)$. For the same reason $\lambda_{1}\left(M, d \tilde{s}^{2}\right)>0$ implies $\lambda_{1}\left(M, d s^{2}\right)>0$. Then by applying Theorem 1.2 , we obtain a smooth function $u$ on $M$ so that $u+f$ is a positive, bounded harmonic function on $\left(M, d s^{2}\right)$. Moreover, we also have $u \in L^{p}\left(M, d s^{2}\right)$. Now all we need to do is to show $u(x) \rightarrow 0$ as $x \rightarrow \infty$. This follows from Theorem 2.2 and that $\left(M, d \tilde{s}^{2}\right)$ admits a global Sobolev constant because $\left(M, d s^{2}\right)$ does. This finishes the proof of the theorem. 


\section{Dirichlet Problem at Infinity}

In Section 2 we studied the boundary value at infinity. In this section we will use the results in Section 1 and Section 2 to solve the Dirichlet problem at infinity for nonpositively curved manifolds with a local pinching condition. All we need to do is to show that boundary data can be achieved by some function $f$ on $M$ so that $|\nabla f|$ is bounded and in $L^{p}$ for some $p \geq 2$.

Theorem 3.1. Let $M$ be an n-dimensional complete, simply-connected manifold with nonpositive sectional curvatures and $\lambda_{1}(M)>0$. Suppose there exist a point $x_{0} \in M$ and a constant $C \geq 1$, such that at any $x \in M$ we have $|K(\sigma)| \leq C\left|K\left(\sigma^{\prime}\right)\right|$, where $\sigma, \sigma^{\prime}$ are plane sections at $x$ containing the tangent vector of the geodesic joining $x_{0}$ to $x$. Then for any continuous function $f$ on $M(\infty)$, there exists $u \in C^{\infty}(M) \cap C^{0}(M \cup M(\infty))$ so that

$$
\begin{cases}\Delta u=0 & \text { on } M, \\ u=f & \text { on } M(\infty) .\end{cases}
$$

Proof. The first step of our proof is to construct a function $f$ on $M$ such that $|\nabla f|$ is bounded and is in $L^{p}$ for some large $p$. We'll consider smooth functions $f$ on $M$ such that outside $B\left(x_{0}, 1 / 2\right)$ it is radially constant with respect to $x_{0}$. We know that $|\nabla f|$ decays uniformly to zero. However, this is not enough to show that $|\nabla f|$ is in $L^{p}$ because there is no assumption on the lower bound of the sectional curvatures. The growth of $|\nabla f|$ has to compare with the volume element in normal coordinates with respect to $x_{0}$.

For any $x \in M$, let $\gamma(t)$ be the unit speed geodesic joining $x_{0}$ to $x$ such that $\gamma(0)=x_{0}$ and $\gamma(r)=x$. Define

$$
F=\left\{V(t) \text { a Jacobi field on } \gamma: V(0)=0,\left\|V^{\prime}(0)\right\|=1, V^{\prime}(0) \perp \gamma^{\prime}(0)\right\} .
$$

Then let $J(x)$ be the supremum of $\|V(r)\|$ for any $V \in F$ and $j(x)$ be the infimum of $\|V(r)\|$ for any $V \in F$. Clearly, in terms of the normal coordinates at $x_{0}$, we see that the volume element at $x$ is bounded from above by $J(x)^{n-1} d r d \theta$, where $d \theta$ is the standard measure on the unit sphere $S^{n-1}$. Also $|\nabla f|(x)$ is bounded from above by $A j(x)^{-1}$, where $A$ is a constant independent of $x$. Therefore, $|\nabla f|^{p}$ will be integrable if $j(x)^{-p} J(x)^{n-1}$ is integrable w.r.t. 
$d r d \theta$. This will follow from the following lemma which is derived in the same way as the Rauch Comparison Theorem.

Lemma 3.2. Let $M, M_{0}$ be Riemannian manifolds and let $\gamma, \gamma_{0}:[0, l] \rightarrow$ $M, M_{0}$ be unit speed geodesics. For each $t \in[0, l]$ and any $X \in M_{\gamma(t)}$, $X_{0} \in\left(M_{0}\right)_{\gamma_{0}(t)}$, let $K(\sigma), K_{0}\left(\sigma_{0}\right)$ denote the sectional curvatures of the plane sections spanned by $X, \gamma^{\prime}(t)$ and $X_{0}, \gamma_{0}^{\prime}(t)$ respectively. Assume that (i) $K(\sigma)$, $K_{0}\left(\sigma_{0}\right) \leq 0$, (ii) $\left|K_{0}\left(\sigma_{0}\right)\right| \leq C|K(\sigma)|$, where $C \geq 1$ is a constant independent of $t, \sigma, \sigma_{0}$. Let $V, V_{0}$ be Jacobi fields along $\gamma, \gamma_{0}$ such thet $V(0)=V_{0}=0$, $V^{\prime}(0) \perp \gamma^{\prime}(0), V_{0}^{\prime}(0) \perp \gamma_{0}^{\prime}(0)$, and $\left\|V^{\prime}(0)\right\|=\left\|V_{0}^{\prime}(0)\right\|$. Then, $\|V(t)\|^{C} /\left\|V_{0}(t)\right\|$ is an increasing function of $t$.

Proof. We will follow the proof in [C-E, p.32-35]. Fixed $t_{1} \in[0, l)$ and define vector fields

$$
W_{t_{1}}(t)=\frac{V(t)}{\left\|V\left(t_{1}\right)\right\|}, \quad W_{0 t_{1}}(t)=\frac{V_{0}(t)}{\left\|V_{0}\left(t_{1}\right)\right\|} .
$$

We now estimate $\frac{d}{d t} \log \left(\|W\|^{2} /\left\|W_{0}\right\|^{2}\right)$. This is exactly the same as $\frac{d}{d t} \log \left(\|V\|^{2} /\left\|V_{0}\right\|^{2}\right)$. Note that

$$
\frac{d}{d t}\left(\log \|W\|^{2}\right)=\frac{2\left\langle V, V^{\prime}\right\rangle}{\langle V, V\rangle} \text { and } \frac{d}{d t}\left(\log \left\|W_{0}\right\|^{2}\right)=\frac{2\left\langle V_{0}, V_{0}^{\prime}\right\rangle}{\left\langle V_{0}, V_{0}\right\rangle} .
$$

Now as $\left\|W\left(t_{1}\right)\right\|=1$, we have at $t_{1}$,

$$
\frac{d}{d t}\left(\log \|W\|^{2}\right)=2 \int_{0}^{t_{1}}\left\langle W, W^{\prime}\right\rangle^{\prime}=2\left(\int_{0}^{t_{1}}\left\langle W^{\prime}, W^{\prime}\right\rangle-K(\sigma)\|W\|^{2}\right)
$$

and there is a similar formula for $W_{0}$. Now as in [C-E, p.33] we transplant $W$ to a vector field $\hat{W}_{0}$ along $\gamma_{0}$ in $M_{0}$. Let $\hat{\sigma}$ be the plane section spanned by $\hat{W}$ and $\gamma_{0}^{\prime}$. Then,

$$
\begin{aligned}
& \frac{1}{2} \frac{d}{d t}\left(\log \frac{\|W\|^{2 C}}{\left\|W_{0}\right\|^{2}}\right) \\
= & C\left(\int_{0}^{t_{1}}\left\langle W^{\prime}, W^{\prime}\right\rangle-K(\sigma)\|W\|^{2}\right)-\int_{0}^{t_{1}}\left\langle W_{0}^{\prime}, W_{0}^{\prime}\right\rangle-K_{0}\left(\sigma_{0}\right)\left\|W_{0}\right\|^{2} \\
= & C\left(\int_{0}^{t_{1}}\left\langle\hat{W}_{0}^{\prime}, \hat{W}_{0}^{\prime}\right\rangle-K_{0}^{\prime}(\hat{\sigma})\|\hat{W}\|^{2}\right)+C\left(\int_{0}^{t_{1}} K_{0}(\hat{\sigma})\|\hat{W}\|^{2}-K(\sigma)\|W\|^{2}\right) \\
& -\int_{0}^{t_{1}}\left\langle W_{0}^{\prime}, W_{0}^{\prime}\right\rangle-K_{0}\left(\sigma_{0}\right)\left\|W_{0}\right\|^{2}
\end{aligned}
$$




$$
\begin{gathered}
\geq(C-1)\left(\int_{0}^{t_{1}}\left\langle\hat{W}_{0}^{\prime}, \hat{W}_{0}^{\prime}\right\rangle-K_{0}(\hat{\sigma})\|\hat{W}\|^{2}\right) \\
+C\left(\int_{0}^{t_{1}} K_{0}(\hat{\sigma})\|\hat{W}\|^{2}-K(\sigma)\|W\|^{2}\right) \\
\geq(C-1)\left(\int_{0}^{t_{1}}-K_{0}(\hat{\sigma})\|\hat{W}\|^{2}\right)+C\left(\int_{0}^{t_{1}} K_{0}(\hat{\sigma})\|\hat{W}\|^{2}-K(\sigma)\|W\|^{2}\right)
\end{gathered}
$$

$$
\geq \int_{0}^{t_{1}}\left(K_{0}(\hat{\sigma})-C K(\sigma)\right)\|W\|^{2} \geq 0 .
$$

Inequality (3.1) follows from the Morse Index Theorem and (3.2) follows from the curvature assumptions and $\|\hat{W}\|=\|W\|$. Since $t_{1}$ is arbitrary, this completes the proof of the lemma.

Let's return to the proof of Theorem 3.1. Lemma 3.2 says that

$$
j(x)^{C} \geq C_{1} J(x)
$$

where $C_{1}$ is a constant independent of $x$. Thus,

$$
j(x)^{-p} J(x)^{n-1} \leq C_{1}^{1-n} j(x)^{C(n-1)-p} .
$$

As the curvature is nonpositive, $j(x) \geq r$. Then for large $p, j(x)^{-p} J(x)^{n-1}$ is integrable w.r.t. $d r d \theta$. Hence, we conclude that $|\nabla f| \in L^{p}$.

Applying Theorem 1.2 to $M$ and $f$ we obtain a smooth function $u \in L^{p}(M)$ so that $\Delta(u+f)=0$. As $M$ is assumed to be nonpositively curved, a global Sobolev constant exists. The application of Theorem 2.2 then completes the proof of Theorem 3.1.

Remark 3.1. Choi [C] solved the Dirichlet problem at infinity for rotationally symmetric manifolds with sectional curvatures decay faster than $-A /\left(r^{2} \log r\right)$ outside a compact set. Note that the local pinching condition is automatically satisfied by rotationally symmetric manifolds. We do not assume any decay rate on the curvature. However, we need $\lambda_{1}(M)>0$ which may not be satisfied by manifolds satisfying the curvature decay condition in Choi's paper.

Remark 3.2. Ballmann [B] solved the Dirichlet problem at infinity for nonpositively curved, irreducible, rank one manifolds which admit a compact quotient. 
The proof uses more tools from probability. One may be able to give an alternative proof using techniques in this paper if one understands more about the growth of Jacobi fields on rank one manifolds.

As in the proof of Theorem 2.2 we can also derive the following corollary.

Corollary 3.3. Suppose that $\left(M, d s^{2}\right)$ is the same as in Theorem 3.1 and that $\left(M, d s_{1}{ }^{2}\right)$ is uniformly equivalent to $\left(M, d s^{2}\right)$. Then, on $\left(M, d s_{1}{ }^{2}\right)$ the Dirichlet problem at infinity can be solved.

\section{REFERENCES}

[Anc] Ancona, A., Negatively curved manifolds, elliptic operators, and the Martin boundary. Ann. of Math., 125 (1987), 495-536.

[A] Anderson, M. T., The Dirichlet problem at infinity for manifolds of negative curvature. J. Diff. Geom., 18 (1983), 701-721.

[A-S] Anderson, M. T. and Schoen, R., Positive harmonic functions on complete manifolds of negative curvature. Ann. of Math., 121 (1985), 429-461.

[B] Ballmann, W., On the Dirichlet Problem at Infinity for Manifolds of Nonpositive Curvature. Forum Math. 1 (1989), 201-213.

[C] Choi, H. I., Asymtotic Dirichlet Problems for Harmonic Functions on Riemannian Manifolds. Trans. of Amer. Math. Soc., 281, 2, Feb., 1984.

[C-E] Cheeger, J. and Ebin, D. Comparison Theorems in Riemannian Geometry. North Holland Publishing Company, Amsterdam, 1975.

[S] Sullivan, D., The Dirichlet problem at infinity for a negatively curved manifold. J. Diff. Geom., 18 (1983), 723-732.

[S-Y] Schoen, R. and Yau, S. T., Differential Geometry. (in Chinese) Scientific Press Beijing, 1988.

[Y] Yau, S. T., Harmonic functions on complete Riemannian manifolds. Comm. PureAppl. Math., 28 (1975), 201-208. 